\title{
Dificuldades, medos e expectativas de gestantes no período gravídico
}

\author{
Difficulties, fears and expectations of pregnant women in the pregnancy period \\ Dificultades, miedos y expectativas de mujeres embarazadas en el periodo de embarazo
}

\begin{abstract}
RESUMO
Objetivo: Conhecer as dificuldades, medos e expectativas de gestantes no período gravídico. Método: Trata-se de um estudo descritivo, com abordagem quantitativa, realizado em uma instituição de ensino superior, em um município Paraibano. $A$ amostra foi composta por 20 gestantes. Os dados foram coletados no mês de setembro de 2018, através de um formulário. Posteriormente, foram analisados estatisticamente, de forma descritiva simples, para posterior apresentação em tabelas. 0 estudo respeitou os aspectos éticos preconizados Resultados: Foi visto nas entrevistas, que as gestantes possuem ter medo e inseguranças em relação a gestação ou parto. Predominantemente, as participantes esperam que o bebê nasça saudável e verbalizaram ter um parto tranquilo sem alterações. Conclusão: Sugere-se a unidade de saúde um reforço na realização de ações internas para os próprios pacientes, para evitar qualquer medo e dúvidas em relação à gestação.

Descritores: Gestação; Mulher; Saúde; Cuidado.
\end{abstract}

\section{ABSTRACT}

Objective: to evaluate the factors associated with the search for a specialized screening service for Covid-19 in the hospital, acObjective: To know the difficulties, fears and expectations of pregnant women during pregnancy. Method: This is a descriptive study, with a quantitative approach, carried out in a higher education institution in a municipality of Paraíba. The sample consisted of 20 pregnant women. Data were collected in September $\mathbf{2 0 1 8}$, using a form. Later, they were statistically analyzed for later presentation in tables. The study respected the recommended ethical aspects. Results: Most interviews have fear and insecurities regarding pregnancy or childbirth. Predominantly, the participants expect the baby to be born healthy and they verbalized that they had a smooth delivery without alterations. Conclusion: It is suggested that the health unit reinforces the performance of internal actions for the patients themselves, to avoid any fear and doubts regarding the pregnancy.

Descriptors: Gestation; Women; Health; Caution.

\section{RESUMEN}

Objetivo: Conocer las dificultades, miedos y expectativas de la gestante durante el embarazo. Método: Se trata de un estudio descriptivo, con enfoque cuantitativo, realizado en una institución de educación superior, en un municipio de Paraíba. La muestra estuvo constituida por 20 mujeres embarazadas. Los datos se recopilaron en septiembre de 2018 mediante un formulario. Posteriormente, fueron analizados estadísticamente para su posterior presentación en tablas. El estudio respetó los aspectos éticos recomendados Resultados: La mayoría de las entrevistas presentan miedos e inseguridades con respecto al embarazo o al parto. Predominantemente, los participantes esperan que el bebé nazca sano y verbalizaron que tuvieron un parto suave y sin alteraciones. Conclusión: Se sugiere que la unidad de salud refuerce la realización de acciones internas para las propias pacientes, para evitar miedos y dudas sobre el embarazo.

Descriptores: Gestación; Mujeres; Salud; Precaución.

RECEBIDO EM: 05/08/2021 APROVADO EM: 109/09/2021

\section{JÉSSICA CRISTINA BEZERRA}

Enfermeira.

ORCID: 0000-0002-9625-8350 


\title{
artigo
}

Bezerra, J C., Costa, E. S, Coêlho, A. F. F. M., Candeia, R. M. S., Santos, J. S., Braga, L. S

Dificuldades, medos e expectativas de gestantes no período gravídico

\section{ERLÂNIA SOUZA COSTA}

Enfermeira. Pós-graduanda em obstetrícia e neonatologia. Faculdade de Enfermagem Nova Esperança.

ORCID: 0000-0001-8185-9471.

\author{
ANA FLÁVIA FREITAS DE MIRANDA COÊLHO \\ Enfermeira. Centro Universitário de João Pessoa. \\ ORCID: 0000-0003-2583-9235.
}

\section{ROZILEIDE MARTINS SIMÕES CANDEIA}

Enfermeira. Mestra em Modelos de Decisão e Saúde - UFPB. Docente Assistente do curso de enfermagem do Centro Universitário de João Pessoa.

ORCID: 0000-0002-2641-1620.

\section{JAYLANE DA SILVA SANTOS}

Enfermeira. Mestranda pelo Programa de Pós-graduação em Modelos de Decisão e Saúde - UFPB.

OCIRD: 0000-0001-8084-7700.

\section{LUANNA SILVA BRAGA}

Enfermeira. Mestre em Enfermagem pelo Programa de Pós-Graduação em Enfermagem da Universidade Federal da Paraíba (PPGENF/UFPB).

ORCID: 0000-0002-0093-0406

\section{INTRODUÇÃO}

A gestação representa o evento de vida mais complexo de uma mulher, podendo ser um momento bastante estressante que produz alterações biológicas, influenciadas pela carga genética, psicológicas e pela estrutura social da mãe (1-2). As alterações fisiológicas observadas durante a gravidez ocorrem basicamente de fatores hormonais voluntárias e involuntárias, que apesar destas modificaçōes serem consideradas normais, configuram-se como adaptações do organismo materno ao ciclo gestacional até o ciclo puerperal (3-4).

Inúmeras investigaçōes na área da saúde apontam que uma relação materno-fetal amorosa e saudável resulta numa vinculação pós-natal eficaz entre mãe e neonato, sendo decorrente dos aspectos emocionais e psíquicos vivenciados por ela, afetando o filho no âmbito neurocomportamental, e influenciando positivamente ou negativamente o concepto(5-6).

De acordo com o pensamento de Costa(7), o ser humano mulher é constituído pelo ciclo vital privilegiado por diversas fases, e podendo desfrutar a fase excepcional de fenômenos fisiológicos que evoluem para a criação de um novo ser, que é a gravidez. Esse momento pode ser considerado a melhor fase de uma mulher com período de mudanças físicas e psicológicas. Esperar um filho, principalmente, o primeiro, é um dos acontecimentos mais importantes da vida de uma mulher, quando relacionado ao desejo da maternidade. Entretanto, o medo de que esse desejo possa ser interrompido causa sentimentos negativos como tristeza, raiva, ansiedade, desapontamento e frustração, podendo desencadear depressões no período gestacional(8).

Segundo Vieira(1), a gravidez além de retratar o período gestacional da evolução embrião/feto, representa também o período do desenvolvimento do papel materno, no qual a gestante passa para uma nova realidade preparando para se tornar mãe. Entre os fatores de risco relacionados as alterações psicológicas na gestação, os conflitos entre estar solteira, casada, divorciada, sem emprego, apresentando pouco apoio social, não tendo suporte emocional, bem como a solidão são os principais fatores para causar angústias e medos à gestante(4-5-6-7) Nesse contexto, o enfermeiro da atenção primária à saúde deve apoiar a mulher ao descobrir a gestação, mostrando à família a importância desse apoio, visto que a falta do mesmo pode gerar consequências negativas para o bem-estar da mulher e do ser humano que está em seu ventre(6-7).

Oliveira, Barbosa, Melo(9) afirmam que uma atenção no pré-natal de qualidade é aquela que tem o início precoce, frequente e com ampla cobertura, onde o enfermeiro deve realizar consultas passando informações sobre a alimentação ideal, cuidado com os mamas para o aleitamento materno, identificação de sinais de parto, assim como assuntos relacionados com a sexualidade na gestação, atividades físicas, primeiros cuidados com o recém-nascido, esclarecendo dúvidas e solucionando possíveis dificuldades que a futura mãe gestante possa apresentar.

Ainda sob a óptica do autor supracitado, o profissional de enfermagem, por sua vez, deve ofertar um suporte psicológico à gestante, estimulando o vínculo familiar, através de conversas claras mostrando os cuidados necessários para o sucesso da gestação, sem julgamentos ou qualquer tipo de preconceito. Esse cuidado facilita que a mulher compartilhe suas fantasias, medos, emoções, amores ou desamores, criando assim um elo de confiança com o enfermeiro, sendo possível haver uma otimização na 
Esperar um filho,

principalmente, $o$

primeiro, é um dos

acontecimentos

mais importantes da

vida de uma mulher,

quando relacionado

ao desejo da

maternidade. assistência de saúde durante o período gravídico. Dessa forma, questionou-se: Quais as dificuldades, medos e expectativas das gestantes no período gravídico? Tem-se por objetivo: Conhecer as dificuldades, medos e expectativas de gestantes no período gravídico.

\section{MÉTODO}

Conceitua-se este estudo como uma pesquisa de campo, de caráter descritivo com abordagem quantitativa. A presente pesquisa foi realizada em uma instituição de ensino superior, no estado da Paraíba, ano de 2018. Nesta pesquisa, a população foi composta por todas as gestantes que realizam o pré-natal na instituição, perfazendo um total de 50 gestantes, porém, a amostra foi constituída por 20 grávidas que foram escolhidas aleatoriamente, levando-se em consideração os seguintes critérios de inclusão: que aceitassem participar livremente do estudo, ser maior de idade e assinar o Termo de Consentimento Livre e Esclarecido (TCLE). Contudo, excluindo assim da pesquisa, as grávidas, por algum motivo se recusem a participar do estudo, não estivessem na unidade no dia da coleta de dados, ou não realizem o pré-natal na referida unidade de saúde.

O instrumento escolhido para a coleta de dados foi um formulário de entrevista, construído pela autora, com nove questões e estruturado, que se caracteriza em duas partes: dados para caracterização socioeconômica da amostra e questões norteadoras acerca do tema em estudo.

As primeiras quatro questões versavam sobre dados de caracterização das participantes, e as demais sobre o tema de forma especifica, que eram: Passou ou passa por alguma dificuldade após a descoberta da gravidez? Possui algum medo em relação a gestação, ao seu filho ou seu parto? Sente-se segura em relação a gestação? Sente-se segura em relação ao parto? Quais são suas expectativas para sua gestação e parto e o seu filho(a)?

De acordo com Neto(10), a coleta de dados determina a adoção de técnicas específicas com fim de conseguir os dados ne- cessários, por meio de pesquisas que foram desenvolvidas com o ofício de questionário, contendo duas partes: dados para caracterização da amostra e questões norteadoras acerca do tema em estudo.

A coleta de dados foi formalizada mediante a aprovação pelo Comitê de Ética em Pesquisa da Faculdade de Enfermagem Nova Esperança, além do encaminhamento de ofício da Coordenação do Curso para a Instituição, local da pesquisa, comunicando a pretensão da mesma.

A pesquisa foi realizada em dias úteis nos turnos manhã e tarde, durante o mês de setembro de 2018, quando foram realizados dois momentos: o primeiro é o contato prévio com as participantes, quando foram passados os objetivos da pesquisa, a sua importância na mesma, e a apresentação do TCLE, o qual foi assinado. O segundo momento é a realização da pesquisa, quando foi esclarecida a finalidade do estudo, garantia do anonimato e procedimento para coleta, ficando os pesquisadores à disposição da entrevistada para quaisquer dúvidas sobre a pesquisa.

O material coletado foi selecionado e analisado com base no enfoque do método quantitativo a partir de dados primários coletados de informações contidas no instrumento de coleta. Posteriormente, os dados foram agrupados através de software Statistical Package for the Social Sciences, guiado pela estatística descritiva simples, para posterior apresentação em gráficos $\mathrm{e}$ tabelas, servindo assim para discussão dos resultados à luz da literatura pertinente. $\mathrm{O}$ presente estudo respeitou os aspectos éticos preconizados pela Resolução CNS 466/12, sob o parecer de $n^{\circ} 183 / 2018$ e CAAE: 97530718400005179 .

\section{RESULTADOS}

Os resultados foram organizados em tabelas e gráficos, divididos em dois momentos para exposição, no qual inicialmente, houve a caracterização da amostra e em seguida, de forma mais minuciosa os dados referentes ao tema central do estudo.

Dados para caracterização da amostra 


\section{artigo}

Bezerra, J. C., Costa, E. S., Coêlho, A. F. F. M., Candeia, R. M. S., Santos, J. S., Braga, L. S.

Dificuldades, medos e expectativas de gestantes no período gravídico

Foram analisadas as entrevistas de 20 gestantes, nos turnos manhã e tarde. Conforme os resultados demonstrados na tabela 01 , a faixa etária dos participantes da pesquisa indica que $40 \%$ (8) das participantes do estudo são da faixa etária entre 24 a 30 anos, enquanto que $25 \%$ (5) estão inseridos na faixa etária entre 31 a 35 anos.

Em relação à escolaridade, $80 \%$ (16) das participantes do estudo afirmaram ter o ensino médio completo, enquanto que $10 \%$ (2) das gestantes tinham ensino médio incompleto, seguido do mesmo percentual de $10 \%$ (2) aquelas que possuíam o ensino superior completo. No tocante a conjugalidade, $65 \%$ (13) das gestantes são casadas e uma pequena parcela de 3\% (15) afirmaram

\begin{tabular}{|c|c|c|}
\hline \multicolumn{3}{|c|}{ FAIXA ETÁRIA } \\
\hline VARIÁVEIS & N & $\%$ \\
\hline $18-23$ anos & 7 & 35 \\
\hline $24-30$ anos & 8 & 40 \\
\hline $31-35$ anos & 5 & 25 \\
\hline Total & 20 & 100 \\
\hline \multicolumn{3}{|c|}{ ESCOLARIDADE } \\
\hline $\begin{array}{l}\text { Ensino médio } \\
\text { incompleto }\end{array}$ & 2 & 10 \\
\hline $\begin{array}{l}\text { Ensino médio } \\
\text { completo }\end{array}$ & 16 & 80 \\
\hline Superior completo & 2 & 10 \\
\hline Total & 20 & 100 \\
\hline \multicolumn{3}{|c|}{ CONJUGALIDADE } \\
\hline Solteira & 4 & 20 \\
\hline Casada & 13 & 65 \\
\hline União Estável & 3 & 15 \\
\hline Total & 20 & 100 \\
\hline \multicolumn{3}{|c|}{ PRIMEIRA GESTAÇÃO } \\
\hline Sim & 14 & 70 \\
\hline Não & 6 & 30 \\
\hline Total & 20 & 100 \\
\hline
\end{tabular}

ter uma união estável. No que diz respeito a primeira gestação, observa-se que $70 \%$ (14) das entrevistadas responderam afirmativamente.

\section{Questões norteadoras do estudo}

Foram questionados as participantes $\mathrm{da}$ pesquisa se já passaram por alguma dificuldade após a descoberta da gravidez e se possuíam algum medo em relação à gestação ou ao parto. Conforme demonstrado na tabela $02,80 \%$ (16) das participantes da pesquisa informaram que não têm dificuldades na gestação e apenas 20\% (4) informaram que tem dificuldades. Das participantes, $65 \%$ (13) afirmaram ter medo em relação à gestação ou parto, enquanto $35 \%(\mathrm{n}=7)$

\begin{tabular}{|c|c|c|}
\hline \multicolumn{3}{|c|}{ DIFICULDADE NA GESTAÇÃO } \\
\hline SIM & 4 & 20 \\
\hline Não & 16 & 80 \\
\hline \multicolumn{3}{|c|}{ MEDO EM RELAÇÃO Ã GESTAÇÃO } \\
\hline Sim & 13 & 65 \\
\hline Não & 7 & 35 \\
\hline Total & 20 & 100 \\
\hline
\end{tabular}

Fonte: Pesquisa direta, João Pessoa - PB, 2018.

TABELA 03 - Dados referentes à segurança em relação gestação de acordo com os participantes da pesquisa

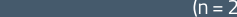

SEGURANÇA EM RELAÇÃO À GESTAÇÃO

\begin{tabular}{|c|c|c|}
\hline SIM & 15 & 75 \\
\hline Não & 5 & 25 \\
\hline
\end{tabular}

SEGURANÇA EM RELAÇÃO AO PARTO

\begin{tabular}{|c|c|c|}
\hline Sim & 12 & 60 \\
\hline Não & 8 & 40 \\
\hline Total & 20 & 100 \\
\hline
\end{tabular}

Eonte: Pesquisa direta $10 a 0_{0}$ Pessoa $-\mathrm{PB}, 2018$.

disseram que não.

Conforme a Tabela 3, as participantes da pesquisa revelam ter ou não seguran- ça em relação à gestação e ao parto, onde $75 \%(15)$ responderam afirmativamente, enquanto que $25 \%$ (5) responderam não. Em se tratando de segurança em relação ao parto, $60 \%$ (12) da amostra responderam positivamente, e $40 \%$ (8) das gestantes responderam não, o que leva a uma relação de confiança com a equipe.

O gráfico 1 apresenta as expectativas das gestantes para a gestação, parto e para o filho, onde $64,3 \%$ (18) disseram que o bebê nasça saudável e perfeito, 35,7\% (10) verbalizaram ter um parto tranquilo sem alterações.

\section{DISCUSSÃO}

A idade materna é considerada como um fator de risco para a gestação. Para o Ministério da Saúde(10), gestantes com idade igual ou superior a 35 anos são consideradas tardias, sendo mais vulneráveis a desenvolver complicações durante a gravidez, o que torna a gestação de alto risco. Nos resultados deste estudo, $25 \%$ das gestantes possuem idade de 31 a 35 anos ou mais, encaixando-se nesse processo.

As mulheres estão engravidando com idade mais avançada, e isso envolve uma gama de fatores, destacando-se as condições de vida da mulher e da família, empoderamento, independência, priorização do trabalho e vida financeira, envolvimento político-social, saúde, sexualidade e seus sonhos(11).

De acordo com Rodrigues et al. (12), as mulheres que concluíram o ensino médio apresentam um nível mais elevado de conhecimento, ajudando assim a ter mais cuidado com sua saúde e no entendimento das doenças e dos cuidados passados pelos profissionais de saúde. A participação ativa do companheiro se torna fundamental ao longo do período gravídico, pois essa relação pai-mãe-feto constrói intimamente um vínculo de afeto um com o outro, motivando a fornecer apoio material e emocional à gestante e desenvolver a paternidade antes da chegada do bebê. Assim, podendo ser decisiva na interação pai-filho, após o nascimento(13).

O parto é um momento esperado que 
GRÁFICO 01 - Representação das respostas dos participantes ao seguinte questionamento: Quais as expectativas para o parto e o filho?

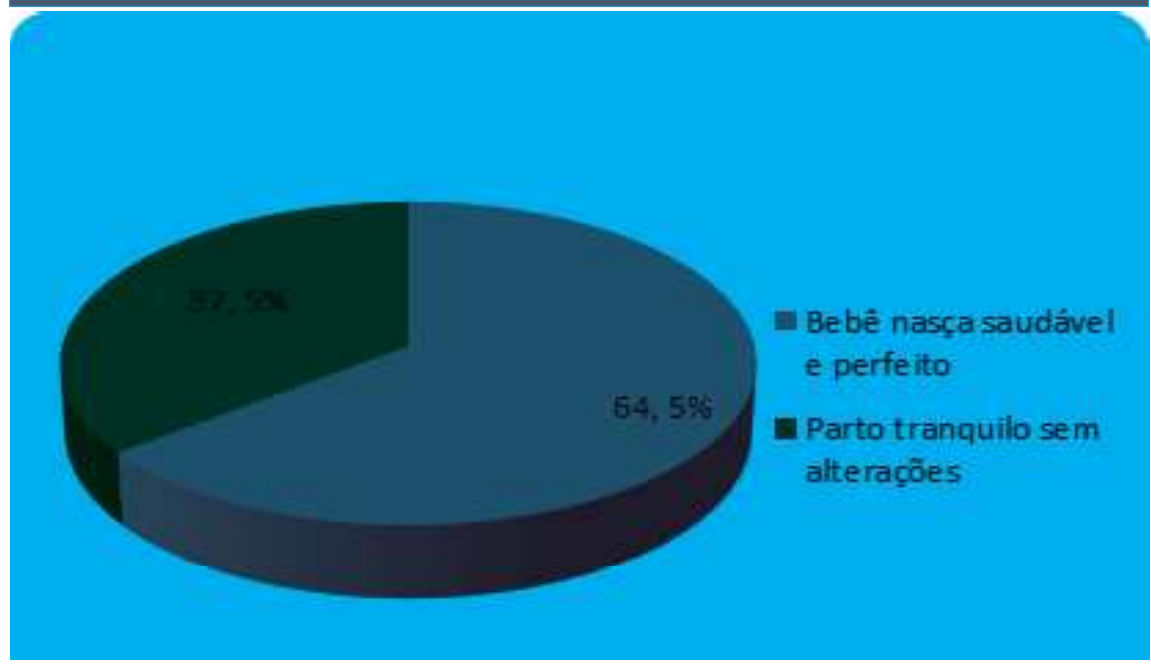

Fonte: Pesquisa direta, João Pessoa - PB, 2018.

vai sendo construído e reconstruído na cultura em que se enquadra as gestantes, como também o parto se torna um momento crítico para maioria delas que vivenciam o trabalho de parto e parto, onde elas associam aos termos de agonia, provação, medo, terror, sofrimento e até a morte. Outro momento que aumenta a ansiedade e o medo é a impossibilidade de dominar o processo, influenciando a incidência de cesariana, juntamente com a ideia de que muitas mulheres ainda sentem medo do parto normal por temerem as consequências que podem advir desta via de parto e sobretudo trazer riscos para o feto ou mesmo provocar lesões anatômicas e fisiológicas(14).

Este anseio pode ser devido ao desconhecimento do que pode vir a ocorrer e pela ausência de informações ou diálogo com os profissionais de saúde quando acompanhada no pré-natal, que não deixam bem esclarecidos as dúvidas em relação ao momento e ao tipo de parto. A gravidez é um período de situações de vulnerabilidades para mulher, seja por fatores de ordem biológica e/ ou contextuais que podem acarretar em riscos e danos para a mãe e o feto, provocando alterações psicológicas, hormonais e físicas. Trazendo condições precárias de moradia e a exposição excessiva a diferentes formas de violência de ordem racial, de gênero, física, sexual, econômica, psicológica, moral e/ou gestação e o parto, mostrando que o profissional de enfermagem não quer ser o sujeito do evento, favorecendo que a mulher e o filho sejam os protagonistas(19).

As expectativas das gestantes para o momento do parto ou do bebê originam-se de seu próprio mundo interno carregado de desejo de um parto seguro, tranquilo e emocionante. Considerando também com as expectativas e sentimentos que têm acerca do seu bebê, a gestante tem as preocupações quanto ao seu desenvolvimento que está relacionado ao sexo, características físicas, psicológicas, interação mãe-bebê e a saúde do bebê(19).

A partir das novas experiências advindas da gestação e do desenvolvimento do papel de mãe, as gestantes podem criar expectativas para o momento do nascimento de seus filhos. Tornar-se mãe não ocorre como uma consequência natural da gestação e do parto $(18,19)$. É uma construção na vida da mulher, repleta de experiências e adaptações diante de mudanças físicas, questões hormonais e novidades que aparecem nesse período. Todas as expectativas e incertezas a respeito do filho, e também da gestação, podem gerar na mulher ansiedade frente ao desconhecido, seja a primeira ou a quinta gravidez, já que cada gestação é única e diferente das outras $(18,19)$. A educação em saúde é uma alternativa para melhorar as expectativas e reduzir os medos, dificuldades e anseios das gestantes, além de prepará-las para o parto e pós-parto(20).

\section{CONCLUSÃO}

As dificuldades, medos e expectativas de gestantes no período gravídico como tema principal deste estudo, viabilizou que foi possível sancionar a verossimilidade com os objetivos outrora propostos. Nesta perspectiva, é possível salientar que os mesmos foram atingidos, através do formulário realizado a fim de colher o maior número de informações importantes.

Ressaltando que a investigação tem uma grande valia na identificação da amostra, pois analisa o perfil da gestante e possibilita o planejamento de ações de promoção e prevenção de saúde, visto que facilita no 


\section{artigo}

Bezerra, J. C., Costa, E. S, Coêlho, A. F. F. M., Candeia, R. M. S., Santos, J. S., Braga, L. S

Dificuldades, medos e expectativas de gestantes no período gravídico

processo de comunicação devido às informaçōes colhidas estarem ligadas a aspectos sociais, culturais e pessoais sobre conhecimentos específicos.

A partir desses dados, sugere-se a unidade de saúde um reforço na realização de açōes internas para os próprios pacientes, para evitar qualquer medo e dúvidas em relação à gestação e seu concepto, como também trazendo à tona a importância das informaçōes concretas, como sendo um dos focos principais, na perspectiva de favore- cer um cuidado integral a gestantes.

Espera-se que este estudo estimule aos participantes da pesquisa, atuantes da Unidade Básica de Saúde, a enfrentar as expectativas durante a gestação acabando com o tabu, levando a buscarem um aprofundamento de conhecimento, com escopo para a melhoria da qualidade de vida relacionada a mãe e bebê, não só a enfermagem, mas sim, todos os profissionais da saúde com um só propósito, o bem-estar dos seus pacientes.

\section{REFERÊNCIAS}

1. Vieira BD, Parizotto APAV. Alterações psicológicas decorrentes do período gravídico. Unoesc \& Ciência - ACBS. Joaçaba, v. 4, n. 1, p. 79-90, jan.-jun. 2013.

2. Arik RM. et al. Percepções e expectativas de gestantes sobre o tipo de parto. Revista Brasileira de Enfermagem. 72, 3, 2019

3. Almeida ARV, Alcântara DS de, Araújo TT de. Expectativas da gestante em relação ao parto. v. 11, n.1. 2018.

4. ação ao bebê. 2017.

5. Silva LS. et al. Análise das mudanças fisiológicas durante a gestação: desvendando mitos. Revista Faculdade Montes Belos (FMB). v. 8, n. 1, p. 1-16, 2015.

6. Andrade CJ, Baccelli MS, Benincasa M. O vínculo mãe-bebê no período de puerpério: uma análise winnicottiana - Revista do NESME., v. 14, n. 1, 2017

7. Costa ES. et al. Alterações Fisiológicas Na Percepção De Mulheres Durante A Gestação. Rev. Rene. Fortaleza, v. 11, n. 2, p. 8693, abr.-jun., 2010.

8. Camarneiro APF, Maciel JCSC, Silveira RMG. Vivências da interrupção espontânea da gravidez em primigestas no primeiro trimestre gestacional: um estudo fenomenológico. Revista de Enfermagem Referência. n. 5,p. 109-117, 2015.

9. Oliveira EC, Barbosa SM, Melo SEP. A importância do acompanhamento pré-natal realizado por enfermeiros. Revista Científica FacMais, v. 7, n. 3. 2016.

10. Brasil. Ministério da Saúde; Secretaria de Atenção à Saúde, Departamento de Ações Programáticas Estratégicas. Gestação de alto risco: manual técnico. Brasília: MS; 2012.

11. Rocha LFA. et al. Significados Nas Representações De Mulheres Que Engravidaram Após Os 35 Anos De Idade. Rev enferm UFPE on line. Recife, v. 8, n. 1, p. 30-36. J an., 2014.

12. Rodrigues Coelho, DD. et al. Gravidez e maternidade tardia: sentimentos e vivências de mulheres em uma unidade de pré-natal de alto risco em barreiras, bahia. Revista das Ciências da Saúde do Oeste Baiano. v. 2, n. 1, p. 01-19. 2017.

13. Martello NV. et al. Percepções de gestantes acerca da participação do companheiro na gestação. Saúde (Santa Maria). v. 43, n. 2, p. 21-2., 2017.

14. Tostes NA, Seidl EMF. Expectativas de Gestantes sobre o Parto e suas Percepções acerca da Preparação para o Parto. Temas em Psicologia. v. 24, n. 2, P. 681-693. 2016.

15. Porto NP. Características Sociodemográficas e de Saúde de Gestantes como Indicadores de Vulnerabilidades. Salvador, 2016. 18f. Trabalho de conclusão de curso (TCC) (Pós-Graduação em Enfermagem Obstétrica) - Escola Bahiana De Medicina E Saúde Pública, Salvador - BA, 2016

16. Leite MG. et al. Sentimentos advindos da maternidade: revelações de um grupo de gestantes. Psicologia em Estudo. Maringá, v. 19, n. 1, p. 115-124, jan.-mar. 2014.

17. Brasil. Humanização do parto. 2002.

18. Ribeiro JF. et al. Contribuição do pré-natal para o parto normal na concepção do enfermeiro da estratégia saúde da família. R. Interd. v. 9, n. 1, p. 161-170, 2016

19. Gomes AG. et al. Expectativas e Sentimentos de Gestantes Solteiras em Relação aos seus Bebês. Temas em Psicologia. v. 23, n. 2, p. 399-411. 2015.

20. Peixoto IVP. A importância da educação em saúde para as gestantes durante 0 acompanhamento do ciclo gravídico puerperal. Revista de Saúde Coletiva de Baueri. v.10, n.57, 2020. 